\title{
Aproximación cualitativa a la Fibromialgia: Aspectos clínicos sobre la atención sanitaria psicológica y transdisciplinar en Costa Rica
}

\author{
Qualitative Approach to Fibromyalgia: Clinical Aspects of \\ Psychological and Transdisciplinary Health Care in Costa Rica
}

Ana Lucía Rodríguez-Gutiérrez ${ }^{1}$

\begin{abstract}
RESUMEN
Este estudio aborda una recopilación de información acerca de distintas variables clínicas sobre la condición reumática-crónica denominada Fibromialgia, tanto en su definición, desde diferentes perspectivas, como en sus posibles causas, su sintomatología, su evaluación diagnóstica, la comorbilidad identificada con otros padecimientos, el tratamiento farmacológico y no-farmacológico comúnmente indicado, las consecuencias de su padecimiento, y su atención sanitaria en el contexto costarricense. Los resultados y el análisis por categorías reúnen la información recopilada de tres entrevistas semiestructuradas, aplicadas a un médico especialista en reumatología, a un psicólogo que trabaja con pacientes con fibromialgia, y a una paciente que padece la enfermedad, diagnosticada once años atrás. Los principales resultados del estudio coinciden con la literatura revisada, aunque se recomienda realizar indagaciones con muestras más grandes para develar la situación epidemiológica nacional de la enfermedad.
\end{abstract}

Palabras Clave: Psicología Clínica y de la Salud, Fibromialgia, sintomatología, comorbilidad, tratamiento.

\begin{abstract}
This study addresses a compilation of information on different clinical variables on the rheumatic-chronic condition called fibromyalgia, both in its definition, from different perspectives, as well as its possible causes, its symptoms, its diagnostic evaluation, the comorbidity identified with other diseases, the treatment pharmacological and non-pharmacological commonly indicated, the consequences of his condition, and his health care in the Costa Rican context. The results and the analysis by categories bring together the information gathered from three semi-structured interviews applied to a specialist in rheumatology, a psychologist who works with patients with fibromyalgia, and a patient with a diagnosis of eleven years of suffering from the disease. The main results of the study coincide with the literature reviewed, although it is recommended to carry out research with larger samples to reveal the national epidemiological situation of the disease.
\end{abstract}

Key Words: Clinical and Health Psychology, Fibromyalgia, Symptomatology, Comorbidity, Treatment.

\footnotetext{
${ }^{1}$ Universidad de Costa Rica. San José, Costa Rica. Bach. en Psicología y Bach. en Enseñanza de la Psicología. Correo electrónico: ana.rodriguezgutierrez@ucr.ac.cr ORCID: https://orcid.org/0000-00023636-9577 DOI: 10.15517/WL.V16I1.47214
}

Recepción: 6/12/2020 Aceptación: 24/5/2021 


\section{Introducción y Antecedentes}

La fibromialgia se considera un desorden crónico, causado por anormalidades neurobiológicas, y caracterizado por dolor fisiológico, fatiga y disfuncionalidad cognitiva. Si bien se sospecha que sus causas se deben a anormalidades neurobiológicas del sistema nervioso central, factores psicológicos, ambientales (sociales) y genéticos, la evidencia indica que la interacción de factores ambientales con los genes asociados a los síndromes de funcionalidad somática y depresión aumentan el riesgo de padecimiento. A pesar de esto, aún no existe un consenso científico sobre la exactitud de su origen (Pereanu 2014).

Más específicamente, el estudio de Geenen y Bijlsma (2010) propone un modelo en donde las alteraciones de funcionamiento hipotalámico-pituitario-adrenal, la hiporreactividad del sistema nervioso autónomo en respuesta a estresores, y cambios estructurales y funcionales en el cerebro juegan un papel crucial en la fibromialgia. En síntesis, se ha observado una "respuesta exagerada de la corticotropina (ACTH) a la hormona liberadora de corticotropina exógena ( $\mathrm{CRH})$ [que] sugiere una hipoactividad crónica de las neuronas CRH y, como consecuencia, hiperactividad de los receptores pituitarios de CRH” (99-100). De este modo, se presentan los factores psicológicos y sociales estrechamente involucrados a la enfermedad, tales como respuestas de estrés, emociones y cambios conductuales perjudiciales (por ejemplo, la disminución del ejercicio físico) (Geenen y Biljsma 2010).

En sí, el modelo propone que los factores predisponentes a la enfermedad (vulnerabilidad genética y exposición a traumas) producen una alteración importante en el sistema nociceptivo, incluyendo varios cambios neuroendocrinos (génesis de los síntomas), y predice que, ante intervenciones farmacológicas y psicológicas exitosas, se esperan mejorías neuroendocrinas y, por tanto, alivio de síntomas (Geenen y Biljsma 2010).

Respecto a la sintomatología física, Rodríguez (2002) enumera principalmente los dolores musculares generalizados, las cefaleas (dolores de cabeza, disturbios del sueño, fatiga) y finalmente, la rigidez muscular. De la misma forma, Burke et al. (2012) definen la fibromialgia como una enfermedad crónica con ciertos síntomas, que incluyen dolor generalizado, rigidez, sensibilidad de músculos, tendones y articulaciones, trastornos del sueño y fatiga. Además, se presenta la evidencia sugerente de que la patogenia 
relacionada con la fibromialgia implica la desregulación de vías de regulación del dolor del sistema nervioso.

El diagnóstico médico, en palabras de Rico-Villademoros y Calandre (2014), y según los autores en concordancia a los criterios del American College of Rheumatology, se efectúa ante la presencia de un dolor generalizado, y otra sintomatología que la acompaña, como la fatiga, trastornos cognitivos, sueño no reparador y diversos síntomas somáticos que suelen ser identificados en relación comórbida con el diagnóstico de este padecimiento en particular. Así mismo, se hipotetiza que el dolor de la fibromialgia parece deberse a una amplificación central de los procesos sensoriales, por lo que se le categoriza como un síndrome de sensibilidad central.

En cuanto a comorbilidad, la fibromialgia coexiste con frecuencia y continuidad, con demás síndromes dolorosos crónicos de enfermedad estructural, como la artritis reumatoide, el lupus eritematoso sistémico, la espondilitis anquilosante, la artrosis y la enfermedad inflamatoria gastrointestinal. Además, esta coexistencia parece tener un impacto en estos otros síndromes. En un estudio con más de 20.000 pacientes con enfermedades reumáticas, principalmente pacientes con artritis reumatoide, mostraron que una mayor intensidad de síntomas de fibromialgia se asociaba a una mayor probabilidad de comorbilidad, por discapacidad laboral, hospitalización y mortalidad. Se han comunicado similares hallazgos coexistencia de fibromialgia y su asociación con una sintomatología más aguda y una mayor discapacidad en pacientes con lupus eritematoso sistémico (Rico-Villademoros y Calandre 2014; Ghib et al. 2016).

La literatura muestra que los tratamientos farmacológicos disponibles para la fibromialgia se centran en antidepresivos tricíclicos, tales como la amitriptilina, y la ciclobenzaprina; o antidepresivos, especialmente la fluoxetina y duloxetina. También, las personas con fibromialgia se tratan mediante analgésicos como el paracetamol, solamente asociado con tramadol, y además, algunos anticonvulsivantes del tipo de la pregabalina. Estos medicamentos mencionados anteriormente, son los que han demostrado mayor eficacia en el control de la sintomatología de la enfermedad.

Además, se está considerando que los antidepresivos inhibidores de la recaptación de serotonina y norepinefrina pueden funcionar como una alternativa en el tratamiento futuro de pacientes con la enfermedad de fibromialgia. En cuanto a los opioides mayores, como la morfina y la oxicodona, por vía oral; o bien, la buprenorfina y el fentanilo en forma de parches transdérmicos, son fármacos cada vez más usados como tratamiento del dolor crónico no oncológico con buenos resultados. Sin embargo, a pesar de poseer un 
buen grado de seguridad en su manejo y un buen perfil terapéutico, estos fármacos son poco utilizados en el tratamiento de la fibromialgia y existen pocos ensayos clínicos que avalen su eficacia en estos pacientes (Rivera 2008; Burke et al. 2012).

A pesar de que la evidencia científica no arroja que exista un proceso inflamatorio de base en este padecimiento, el uso de antiinflamatorios no esteroideos (AINEs) se encuentra ampliamente extendido en el tratamiento de la fibromialgia, ya que se emplean en dosis sub-terapéuticas, en donde se comportan más como analgésicos que como antiinflamatorios propiamente (Rivera 2008).

De igual forma, además de que se ha demostrado gran eficacia del arsenal terapéutico medicamentoso (los antidepresivos clásicos, inhibidores de la recaptación de serotonina y de noradrenalina), existen terapias no farmacológicas, de las cuales, las más utilizadas son la psicoterapia, la fisioterapia, los quiromasajes, acupuntura, los ejercicios aeróbicos de bajo impacto y la terapia de música con vibración (Ubago et al. 2005; Weber et al. 2015).

Por un lado, desde la perspectiva psicológica, Villanueva et al. (2004) proponen que en la población que sufre de fibromialgia, inciden factores psicosociales muy importantes, tales como la convicción de que se trata de una enfermedad seria con fuertes expectativas de empeoramiento, de la mano con una alarma catastrofista y sensacionalista. Asimismo, dentro de la sintomatología figuran alteraciones del sueño, principalmente insomnio, sueño ligero, despertar frecuente, bruxismo y sueño reparador; distrés psicológico con una elevada asociación a ansiedad, disforia, alteraciones del humor y cuadros distímicos, entre otros, trastornos de pánico y de fobia simple.

De igual forma, Revuelta-Evrard, Segura-Escobar y Paulino-Tevar (2010) argumentan que los síntomas afectivos más comunes son la tristeza patológica o el humor depresivo, caracterizados por sentimientos desagradables, disforia, anhedonia, ansiedad, angustia, irritabilidad y apatía. Entre los principales síntomas cognitivos pueden figurar la bradipsiquia (enlentecimiento del pensamiento), una excesiva rumiación y preocupación, pensamientos pesimistas, de desesperanza e inutilidad, de baja autoestima, ideas de muerte y suicidio. También, dentro de los síntomas conductuales, puede presentarse "llanto frecuente e inmotivado, alteraciones de la actividad psicomotora, disminución del rendimiento en los diferentes aspectos de la vida, y tendencia al aislamiento. Las alteraciones más graves son el intento de suicidio y el suicidio consumado (poco frecuentes en fibromialgia)" (Revuelta-Evrard, Segura-Escobar y Paulino-Tevar 2010: 329). 
Gómez-Argüelles y Anciones (2009) encontraron en su estudio, además de los síntomas descritos, sintomatología neuropsicológica, específicamente en problemas de memoria y concentración, además de un importante reporte de cefaleas tensionales, fatiga psíquica y parestesias.

Por otro lado, Villanueva et al. (2004) argumentan que la terapia psicológica mediante la relajación con biofeedback, la terapia cognitivo-conductual y los programas de modificación del comportamiento son las técnicas más empleadas en el abordaje desde esta especialidad, en combinación con la terapia farmacológica prescrita. Por su parte, Pikoff (2010) propone en su estudio que, precisamente, la terapia cognitivo-conductual es la que posee mayor evidencia de efectividad en el tratamiento psicológico de esta enfermedad, mientras que Parra, Latorre y Montañés (2012) añaden que la terapia cognitiva enfocada en Mindfulness ha resultado muy efectiva para la reducción de síntomas de ansiedad en personas con fibromialgia.

En general, en la guía del National Institute for Health and Care Excelence (NICE, 2020b) para la atención clínica del dolor neuropático en adultos, se recomiendan principios claves tales como discutir con la persona paciente sobre la gravedad del dolor y su impacto en el estilo de vida, los problemas físicos y psicológicos subyacentes, los beneficios y efectos secundarios del tratamiento farmacológico empleado, y sobre tratamientos no farmacológicos tales como cirugía, terapias físicas y psicológicas. Respecto a esta última área, se menciona la importancia de ayudar al consultante con estrategias de afrontamiento al dolor y significar un apoyo en su toma de decisiones, así como en su proceso de adherencia al tratamiento. Actualmente, NICE se encuentra desarrollando una guía para la evaluación y el manejo de la fibromialgia tanto desde la óptica médica como desde la psicología (NICE, 2020a).

De acuerdo con lo anterior, Boomershine (2011) indica también la importancia de valorar la intensidad del dolor, la disfuncionalidad física, y la presencia de depresión, ansiedad e hipersensibilidad, que pueden ser evaluados a través de instrumentos específicos (NRS del BPI; FIQR; HADS-D; BDI, HADS-A; y MTPS-FIS, respectivamente).

Con respecto a las consecuencias del padecimiento de la fibromialgia, se ha encontrado que esta enfermedad resta calidad de vida a las personas pacientes, lo que resulta para estas una condición sumamente incapacitante y complicada de sobrellevar. Durante períodos de crisis, cualquier actividad cotidiana puede requerir un gran esfuerzo, si se toma en cuenta aquellas tareas básicas como cepillarse los dientes, o bien, ponerse 
zapatos. La enfermedad afecta la realización de labores domésticas, y se complica, significativamente, la posibilidad de mantener un trabajo o un empleo formal que sea remunerado (Escudero et al. 2010).

Asimismo, los pacientes relatan que dejan de salir, de divertirse y se sienten sin ganas de nada, por lo que tienen repercusiones a nivel social y laboral. Su círculo de apoyo se ve considerablemente reducido, ya que la experiencia de dolor y otros síntomas provoca que las personas pacientes tengan cambios de humor, o bien, de carácter, y que experimenten tensión y nerviosismo, lo cual conlleva a que su día a día sea aún más difícil (Escudero et al. 2010).

Además, este padecimiento se ve agravado considerablemente por la incomprensión que reciben de las personas de su entorno y de su ambiente, más aún cuando carecen de diagnóstico clínico, y por la incomprensión de sus profesionales sanitarios, ya que muchos afirman que sus dolencias son producto de su imaginación, que están locas, mal de los nervios, que somatizan o que son unas histéricas (Escudero et al. 2010), por lo cual, las repercusiones negativas al padecer fibromialgia se conjugan a un estigma social de la enfermedad.

Entre otras consecuencias negativas de padecimiento, se encuentran una alta correlación de sufrir fibromialgia con un daño axonal subclínico del nervio retinal (sugiriendo procesos neurodegenerativos), disfunción sexual masculina, discapacidad laboral y retiro laboral prematuro, problemas financieros, pensamientos suicidas (GarcíaMartin et al. 2016; Batmaz et al. 2013; Sallinen et al. 2010) y un deterioro comórbido de los parámetros clínicos cuando la persona padece simultáneamente del síndrome de fatiga crónica, con una reducción importante en la calidad de vida (Faro et al. 2014).

Respecto a la prevalencia epidemiológica de esta condición, las estadísticas muestran que la fibromialgia afecta aproximadamente a un $2 \%$ de la población estadounidense, con una ocurrencia de diagnóstico ocurre entre los 20 y 50 años de edad, y que el padecimiento se presenta mayoritariamente en mujeres más que en hombres, proporcionalmente en 9:1 (Burke et al. 2012).

En Costa Rica, la falta de investigación epidemiológica no permite tener una visión clara sobre la situación actual del padecimiento, por lo que únicamente se cuenta con los datos de egreso según el diagnóstico principal de la Caja Costarricense del Seguro Social (2017). Se plantea que para enfermedades del sistema osteomuscular y del sistema conjuntivo, se brindó un total de 40405 consultas en el año 2017, dentro del criterio Otros 
del sistema osteomuscular, donde se puede catalogar la fibromialgia entre otros padecimientos.

Dada la importancia de investigación en el tema, tanto desde el área de la Psicología Clínica y de la Salud, como por la falta de estudios a nivel nacional, el objetivo general del presente estudio es hacer una indagación exploratoria, a través de entrevistas a profesionales de la salud y quien padece la enfermedad, de los principales focos clínicos de la fibromialgia contextualizados a la realidad costarricense.

\section{Método}

\subsection{Participantes}

Se contactó a tres participantes para el estudio por medio de comunicación telefónica, gracias a la referencia de otros profesionales conocidos. La primera participante fue una mujer de cincuenta y dos años, costarricense, cuya trayectoria de vida fue marcada por el diagnóstico de artritis reumatoide en el año 2005. A pesar de que su enfermedad comenzó a ser tratada farmacológicamente por el médico reumatólogo, la paciente continuó experimentando dolor osteomuscular no localizado, por lo que, tras varias pruebas, dos años después fue diagnosticada con fibromialgia por el especialista. Actualmente, continúa controlando la artritis reumatoide y la fibromialgia mediante tratamiento farmacológico y reducción del estrés, principalmente.

El segundo participante de la investigación fue un profesional en Psicología de la Universidad de Costa Rica. El psicólogo tuvo experiencia trabajando en el tratamiento psicosocial de personas con fibromialgia en la Clínica del Dolor, perteneciente al Hospital Calderón Guardia de la Caja Costarricense del Seguro Social, gracias a su tema de trabajo final de graduación, que consistió en una práctica dirigida bajo campo clínico ${ }^{2}$.

El último participante entrevistado fue un especialista reumatólogo que trabaja en el servicio de Reumatología del Hospital Calderón Guardia brindando atención pública. Ha ejercido la especialidad durante diez años y tiene amplia experiencia en el diagnóstico y tratamiento de la fibromialgia, ya que una gran parte del público que atiende padece la enfermedad.

\footnotetext{
${ }^{2}$ Ramírez, J., «Inserción profesional en el abordaje psicológico de pacientes con enfermedades crónicas y terminales y sus familiares en el Centro Nacional de Control del Dolor y Cuidado Paliativo de la C.C.S.S.» (Memoria de Práctica Dirigida, Universidad de Costa Rica, 2019).
} 


\subsection{Procedimiento}

Con base en la revisión de literatura, se contemplaron ocho focos de información para la formulación de dos guías de entrevistas semiestructuradas (las preguntas variaron un poco por el tipo de participante, paciente o profesional en salud, pero el núcleo temático se mantuvo): (1) Información personal/profesional, (2) Definición, causas y detonantes de la enfermedad, (3) Sintomatología y comorbilidad, (4) Historia clínica y diagnóstico, (5) Epidemiología, (6) Tratamiento farmacológico / no farmacológico, (7) Tratamiento a nivel público / privado y (8) Consecuencias del padecimiento.

Para la realización de cada entrevista por separado, en un primer momento se solicitó a la persona paciente la lectura y firma de Consentimiento Informado, y a los profesionales en salud su consentimiento oral, dejando en claro a las tres personas implicadas los objetivos investigativos, el acuerdo de grabación en audio de la entrevista y el aseguramiento de su confidencialidad. Seguidamente, se dio inicio con las preguntas respectivas de cada guía y aquellas que los entrevistadores consideraran pertinentes en el curso de la entrevista. Al finalizar, se agradeció por la participación y se mostró apertura a las personas participantes para contactar en caso de que surgieran dudas sobre el estudio.

Una vez llevado a cabo el proceso de entrevista, se hizo transcripción y análisis de todo el material en audio, con el fin de identificar las categorías de información coincidente en las tres entrevistas.

\section{Resultados}

A continuación, se presenta en la Tabla 1 las categorías de información generadas a partir del análisis del material en audio, con sus respectivas subcategorías. 
Tabla 1. Resultados por categorías y subcategorías construidas a partir de los datos de las entrevistas.

\begin{tabular}{|c|c|c|c|c|c|c|c|}
\hline 1. Definición & 2. Causas & 3. Sintomatología & $\begin{array}{l}\text { 4. Evaluación } \\
\text { Diagnóstica }\end{array}$ & 5. Comorbilidad & 6. Tratamiento & $\begin{array}{l}\text { 7. Consecuencias } \\
\text { del Padecimiento }\end{array}$ & $\begin{array}{l}\text { 8. Atención en } \\
\text { Costa Rica }\end{array}$ \\
\hline $\begin{array}{l}\text { Síndrome } \\
\text { crónico de la } \\
\text { percepción del } \\
\text { dolor (con } \\
\text { presencia al } \\
\text { menos de tres } \\
\text { meses), } \\
\text { caracterizado } \\
\text { por malestar y } \\
\text { dolor } \\
\text { generalizado, } \\
\text { principalmente } \\
\text { en áreas } \\
\text { musculo- } \\
\text { esqueléticas y } \\
\text { en } \\
\text { articulaciones. }\end{array}$ & $\begin{array}{l}\text { Orgánicas } \\
\text { *Padecer } \\
\text { enfermedad que } \\
\text { predispone a } \\
\text { dolor crónico. } \\
\text { *Disminución de } \\
\text { receptores sero- } \\
\text { toninérgicos y } \\
\text { norepirenérgicos } \\
\text { *Disminución de } \\
\text { hormona de } \\
\text { crecimiento, lo } \\
\text { cual coadyuva a } \\
\text { la génesis de } \\
\text { microtraumatis- } \\
\text { mos musculares. } \\
\text { *Aparición de la } \\
\text { enfermedad tras } \\
\text { accidente. } \\
\text { *Problemas de } \\
\text { procesamiento } \\
\text { de información }\end{array}$ & $\begin{array}{l}\text { Fisiológica } \\
\text { *Dolor. } \\
\text { *Cansancio, } \\
\text { debilidad, fatiga. } \\
\text { *Insomnio. } \\
\text { /Hipersomnia. } \\
\text { *Disestesias / } \\
\text { Parestesias. } \\
\text { *Cefaleas. } \\
\text { Psicológica } \\
\text { *Cambios del } \\
\text { estado de ánimo. } \\
\text { *Pensamientos } \\
\text { catastróficos, } \\
\text { negativos. } \\
\text { *Ansiedad, } \\
\text { preocupación } \\
\text { excesiva (incide } \\
\text { en los hábitos } \\
\text { alimenticios). } \\
\text { *Apatía. }\end{array}$ & $\begin{array}{l}\text { Médica } \\
\text { *Puntos de } \\
\text { dolor (8 a } 10 \text { li- } \\
\text { bras de presión; } \\
\text { científicamente } \\
\text { descontinuado). } \\
\text { * Sin } \\
\underline{\text { alteraciones en }} \\
\text { la exploración } \\
\text { física reumato- } \\
\text { lógica (color, } \\
\text { temperatura e } \\
\text { inflamación de } \\
\text { área afectada) y } \\
\text { en exámenes de } \\
\text { sangre comu- } \\
\text { nes (hemoglo- } \\
\text { bina, plaquetas, } \\
\text { leucocitos, } \\
\text { función hepáti- } \\
\text { ca y renal) y } \\
\text { reumatológicos }\end{array}$ & $\begin{array}{l}\text { *Artritis } \\
\text { reumatoide. } \\
\text { *Hiperlaxitud } \\
\text { articular. } \\
\text { *Escoliosis. } \\
\text { *Lupus } \\
\text { eritematoso } \\
\text { sistémico. } \\
\text { *Reumatismo de } \\
\text { partes blandas } \\
\text { (malformaciones } \\
\text { físicas que } \\
\text { repercuten en la } \\
\text { columna y } \\
\text { generan dolor } \\
\text { crónico). } \\
\text { *Problemas } \\
\text { gastrointestinales } \\
\text { (náuseas, } \\
\text { gastritis, colitis). } \\
\text { *Trastornos del } \\
\text { ánimo }\end{array}$ & $\begin{array}{l}\text { Farmacológico } \\
\text { Antidepresivos } \\
\text { *Amitriptilina } \\
\text { (tricíclico). } \\
\text { *Fluoxetina - } \\
\text { Duloxetina. } \\
\text { *Inhibidores de } \\
\text { la recaptura de } \\
\text { serotonina y } \\
\text { norepinefrina. } \\
\text { Analgésicos } \\
\text { *Ibuprofeno. } \\
\text { *Acetaminofén. } \\
\text { *Voltarén } \\
\text { inyectable (anti- } \\
\text { inflamatorio no } \\
\text { esteroideo). } \\
\text { No } \\
\text { farmacológico }\end{array}$ & $\begin{array}{l}\text { *Incomprensión } \\
\text { de la } \\
\text { enfermedad, } \\
\text { estigma social. } \\
\text { * Menor calidad } \\
\text { en la atención } \\
\text { sanitaria por } \\
\text { prejuicios sobre } \\
\text { la enfermedad. } \\
\text { *Conflictos } \\
\text { familiares y de } \\
\text { pareja. } \\
\text { *Enfermedad } \\
\text { incapacitante en } \\
\text { el trabajo. } \\
\text { *Indisposición } \\
\text { para hacer } \\
\text { actividades } \\
\text { cotidianas. }\end{array}$ & $\begin{array}{l}\text { Sector público } \\
\text { *Diagnóstico y } \\
\text { prescripción de } \\
\text { fármacos se } \\
\text { hace en servicio } \\
\text { de reumatología. } \\
\text { *La atención se } \\
\text { da una vez al } \\
\text { año. } \\
\text { *Referencia a } \\
\text { médico general } \\
\text { para dar } \\
\text { continuidad a } \\
\text { medicamentos. } \\
\text { *Gran } \\
\text { porcentaje de la } \\
\text { atención es por } \\
\text { fibromialgia } \\
\text { (25\%), y la } \\
\text { mayoría son } \\
\text { mujeres (95\%, } \\
\text { de 30-50 años). }\end{array}$ \\
\hline
\end{tabular}




\begin{tabular}{|c|c|c|c|c|c|}
\hline $\begin{array}{l}\text { Fibromialgia } \\
\text { Tipo 2: Se } \\
\text { presenta } \\
\text { simultánea- } \\
\text { mente con } \\
\text { otra(s) } \\
\text { enferme- } \\
\text { dad(es) } \\
\text { asociada(s). }\end{array}$ & $\begin{array}{l}\text { dolorosa a nivel } \\
\text { de sistema } \\
\text { nervioso central. } \\
\text { (hipersensibili- } \\
\text { dad por exceso } \\
\text { de sustancia P). } \\
\text { Ambientales, } \\
\text { psicosociales } \\
\text { *Alta exposición } \\
\text { a situaciones de } \\
\text { estrés } \\
\text { (problemáticas } \\
\text { durante la } \\
\text { infancia, en el } \\
\text { trabajo, econo- } \\
\text { mía personal, } \\
\text { relaciones } \\
\text { familiares, etc.). }\end{array}$ & $\begin{array}{l}\text { (factor } \\
\text { reumático, } \\
\text { anticuerpo } \\
\text { antipéptido } \\
\text { cíclico (Anti } \\
\text { PPC), } \\
\text { anticuerpos } \\
\text { antinucleares). } \\
\text { Evaluación } \\
\text { Psicológica del } \\
\text { Dolor Total } \\
\text { Se evalúan las } \\
\text { afectaciones } \\
\text { en: } \\
\text { *Área física. } \\
\text { *Área social. } \\
\text { *Área } \\
\text { psicológica. } \\
\text { *Área } \\
\text { espiritual. }\end{array}$ & $\begin{array}{l}\text { (especialmente } \\
\text { depresión). }\end{array}$ & $\begin{array}{l}\text { *Ejercicio } \\
\text { aeróbico de bajo } \\
\text { impacto o } \\
\text { natación } \\
\text { (especialmente } \\
\text { en agua } \\
\text { temperada). } \\
\text { *Terapia física. } \\
\text { *Terapia } \\
\text { psicológica } \\
\text { (psicoeducación } \\
\text { de la enferme- } \\
\text { dad, terapia de } \\
\text { Beck para tratar } \\
\text { las distorsiones } \\
\text { cognitivas, me- } \\
\text { moria del dolor } \\
\text { (cambios en la } \\
\text { interpretación } \\
\text { del dolor), acti- } \\
\text { vación conduc- } \\
\text { tual, adherencia } \\
\text { al tratamiento y } \\
\text { estrategias para } \\
\text { el manejo del } \\
\text { estrés/ansiedad). }\end{array}$ & $\begin{array}{l}\text { *La atención } \\
\text { integral se } \\
\text { maneja en las } \\
\text { clínicas del } \\
\text { dolor } \\
\text { (Psicología - } \\
\text { Medicina - } \\
\text { Terapia Física). } \\
\text { Sector privado } \\
\text { *Uso de } \\
\text { fármacos más } \\
\text { efectivos } \\
\text { (Cymbalta, } \\
\text { Dium). }\end{array}$ \\
\hline
\end{tabular}




\section{Análisis de los resultados por categorías}

\subsection{Definición del padecimiento}

Respecto a esta categoría, se concluye la premisa de que hay diferencias en cuanto a la percepción y noción de la fibromialgia, en relación con el enfoque transdisciplinar, ya que existen una serie de síntomas que la distinguen como una enfermedad propiamente desde un foco médico, y otros que la categorizan como una condición, a partir de una visión psicológica.

En la entrevista realizada al reumatólogo del sector público, este inició conceptualizando la Fibromialgia como una enfermedad, mientras que en la entrevista realizada a la paciente con fibromialgia, ella afirmaba que, si bien inicialmente concebía a la fibromialgia como una enfermedad, con el pasar de los años, ella siguió informándose sobre su propio estado y concluyó que es una condición crónica, por lo cual sabía que no podía curarse, pero sí mejorar sus síntomas. El psicólogo que trabaja con pacientes ya diagnosticados, definió la fibromialgia como una enfermedad de mujeres, ya que se presenta más en mujeres que en hombres. Asimismo, afirmó que es una condición crónica que no tiene cura, cuyas causas son desconocidas

Además, en la entrevista realizada al reumatólogo, se recopiló que existen dos tipos de fibromialgia. La de tipo 1, se caracteriza porque se presenta sola, sin otros padecimientos; la de tipo 2 es una fibromialgia que no se presenta por sí misma, sino que, se da por padecimientos, o bien, enfermedades que se hayan encontrado anteriormente en la persona, es decir, que se presenta de forma simultánea con otras enfermedades asociadas.

\subsection{Causas}

En cuanto a lo que expusieron las personas entrevistadas, con respecto a las causas de la fibromialgia, no se logró descifrar si existe alguna en específico que detone la enfermedad. Sin embargo, las causas que se recopilaron se identificaron en dos subcategorías principalmente, orgánicas y ambientales/psicosociales. Con respecto a esto último, cabe destacar que la paciente, el psicólogo y el reumatólogo, en sus entrevistas respectivas, parecen estar de acuerdo con que el estrés empeora los síntomas de la fibromialgia significativamente, por lo que se halla cierta relación con lo que exponen los teóricos. 


\subsection{Sintomatología}

La paciente diagnosticada con el padecimiento afirmó que experimentaba síntomas tanto físicos como psicológicos, tales como mucho dolor, cansancio, debilidad, dolores de cabeza, cambios en su estado de ánimo, pensamientos catastróficos e invasivos, preocupaciones, ansiedad y estrés. Comentaba que, ante esto, debía optar por relajarse y hacer modificaciones en su estilo de vida. En palabras del psicólogo, la fibromialgia se caracteriza por dolor físico prioritariamente, debido a una disfunción en cuanto a la percepción de la información sensorial, que se traduce en una hipersensibilidad en temas de dolor. A parte de esta información, el reumatólogo añadió que las personas con fibromialgia suelen tener importantes alteraciones en el sueño, en el extremo de dormir demasiado o en el otro extremo de dormir muy poco, así como dolores de cabeza frecuentes, alteraciones en la sensación táctil (disestesias) y hormigueo/adormecimiento de extremidades (parestesias).

\subsection{Evaluación diagnóstica}

Referente al diagnóstico, el psicólogo detalló en la entrevista que los reumatólogos son quienes se encargan de diagnosticar a las y los pacientes de fibromialgia, por su grado de experticia profesional y de conocimiento. Por su parte, el médico argumentó que, para diagnosticar esta enfermedad, el profesional realiza exploraciones físicas reumatológicas, es decir, valora el color de la piel de la persona paciente en áreas con dolor, la temperatura e inflamación del área afectada y los resultados de exámenes de sangre comunes (hemoglobina, plaquetas, leucocitos) y de exámenes reumatológicos (factor reumático, anticuerpos-anti-péptidos-cíclicos: Anti-PPC, y anticuerpos antinucleares). Si la valoración general de dichos factores descarta el diagnóstico de otra enfermedad médica o reumatológica, se considera el diagnóstico del padecimiento en estudio.

Con respecto a la evaluación psicológica, el psicólogo detalló que esta contempla una valoración del dolor total, es decir, la afectación específica que presenta la persona paciente en sus áreas personales: física (escala del dolor, autocuidado, etc.), social (comunicación, socialización, redes de apoyo), psicológica (niveles emocional, cognitivo y conductual) y espiritual (identificación de factores protectores relacionados a la espiritualidad de la persona paciente, o malestar emocional relacionado a su condición y su espiritualidad). 


\subsection{Comorbilidad}

En cuanto a comorbilidad de la fibromialgia, por un lado, el psicólogo afirmó que el estrés y la ansiedad están comúnmente presentes en pacientes con fibromialgia, y que, a su vez, estos influyen en fluctuaciones en los estados anímicos de los y las pacientes, lo cual puede desencadenar en episodios depresivos. Asimismo, la paciente afirmó haber tenido depresión en algún momento de su vida, después de ser diagnosticada con la fibromialgia, lo cual se relaciona directamente con la literatura revisada.

Por otro lado, según las afirmaciones del reumatólogo, la fibromialgia también se asocia a otras enfermedades musculoesqueléticas, en especial a la artritis reumatoide, a la hiperlaxitud articular, a la escoliosis, al lupus eritematoso sistémico, así como a trastornos de sueño y a problemas gastrointestinales, tales como gastritis, colitis y náuseas.

\subsection{Tratamiento}

En la entrevista realizada al médico especializado en reumatología, se encontró que los tratamientos farmacológicos más efectivos son los antidepresivos tricíclicos, y algunos analgésicos.

Por otra parte, el médico reumatólogo y el psicólogo sugirieron como terapias no farmacológicas ni convencionales los ejercicios aeróbicos de bajo impacto, como la natación, pero en aguas temperadas, en palabras de ambos, debido a que favorece a la reducción de la sintomatología de la fibromialgia en sobremanera y que la mayoría de las pacientes tienden a presentar mejorías en múltiples ámbitos, físicos y sociales.

Con respecto a la psicoterapia, el psicólogo explicó que las intervenciones se realizan tomando en cuenta las especificidades de cada paciente, pero que a nivel general se recurre a brindar psicoeducación sobre la fibromialgia y las consecuencias psicológicas que contrae, se brinda apoyo en la adherencia al tratamiento farmacológico y se trabaja con la memoria del dolor, de modo que la persona identifique de una manera más objetiva la percepción de severidad que tiene sobre su malestar cotidianamente. Según el caso, también se puede trabajar con terapia cognitiva para manejar pensamientos catastróficos o disonancias cognitivas importantes que sean perjudiciales para la persona, en conjunción con entrenamiento en diferentes estrategias para el manejo del estrés y la ansiedad. En casos donde la persona presente una desmotivación importante por las actividades de la vida cotidiana o depresión, se trabaja con la activación conductual para que la persona continúe su vida de la manera más adecuada. 


\subsection{Consecuencias psicosociales del padecimiento}

En relación con las consecuencias psicosociales del padecimiento, se llega a un consenso entre las tres entrevistas realizadas, de que el padecimiento de fibromialgia tiene repercusiones a nivel personal, social, familiar y laboral. Asimismo, se presenta la idea por parte de la paciente, del psicólogo y del reumatólogo, de que la fibromialgia es una enfermedad altamente estigmatizada socialmente, y que esto representa una amenaza, tanto para el involucramiento social, como en la calidad de atención clínica para las personas quienes padecen esta enfermedad.

\subsection{Atención en instituciones de salud pública}

En cuanto a la atención pública de la fibromialgia en Costa Rica, según el reumatólogo entrevistado, se brinda atención en dicha especialidad una vez por año, ya realizado el diagnóstico, y se refieren al primer nivel de atención a partir de su diagnóstico: a medicina general para dar continuación y seguimiento al tratamiento farmacológico y a sus medicamentos. De acuerdo con el médico, un gran porcentaje de las personas que se atienden por consulta en el área de reumatología es por fibromialgia, un $25 \%$ del total de consultas, aproximadamente, siendo un $95 \%$ total de esas consultas población femenina.

En el Centro Nacional del Control del Dolor, según el psicólogo entrevistado, se tratan de manera integral, a partir de terapias distintas, con un formato de terapia grupal, con tal de velar por un mejoramiento en la calidad de vida de estas personas; sin embargo, se habla de que hay una gran disfuncionalidad familiar después de que se diagnostican con la enfermedad.

La diferencia del tipo de atención con el área privada en Costa Rica se desconoce, no obstante, el medicamento es el que cambia, según el reumatólogo, ya que en el sector privado en Costa Rica se receta Cymbalta y Dium, los cuales son considerados mayormente eficaces por parte del reumatólogo entrevistado.

\section{Discusión y conclusiones}

En este estudio, fue posible vislumbrar que la fibromialgia es concebida a nivel general como una condición de dolor crónico no localizado, que afecta a un sinnúmero de pacientes tanto a nivel internacional como nacional, principalmente a mujeres (Burke 
et al. 2012). Los resultados de esta investigación coinciden con la falta de consenso sobre la definición del padecimiento como una enfermedad, ya que no se ha identificado una causa exacta sobre su génesis (Pereanu 2014). Sin embargo, los resultados coinciden con la literatura en tanto ciertos factores orgánicos, como la hipersensibilidad a causa de una alteración nerviosa y padecer otra(s) enfermedad(es) asociadas a dolor crónico, así como factores ambientales/psicosociales asociados a la vivencia de situaciones de estrés, son predisponentes a esta condición (Sommer et al. 2012 citado en Pereanu 2014, Geenen y Bijlsma 2010).

Además, se encontró coincidente con la literatura revisada, que la sintomatología no solamente involucra aspectos fisiológicos (Geenen y Bijlsma 2010, Rodríguez 2002, Rico-Villademoros y Calandre 2014, Ghib et al. 2016), sino también cambios psicológicos importantes en la persona que padece fibromialgia, principalmente en fluctuaciones del estado de ánimo, una gran presencia de pensamientos catastróficos asociados a la severidad del dolor experimentado y a su condición de salud, ansiedad, depresión, y la sensación de estigmatización por la consideración sobre el padecimiento como una enfermedad no real (Villanueva et al. 2004; Revuelta-Evrard, Segura-Escobar y Paulino-Tevar 2010; Escudero et al. 2010), lo cual representa consecuencias negativas con graves repercusiones a nivel personal, familiar y social para la persona consultante, lo que agrega a este último espacio la afectación en la calidad de atención clínica que la persona recibe en centros de salud públicos y privados.

Los resultados arrojaron que la evaluación diagnóstica médica es realizada en el contexto nacional por reumatólogos, quienes detectan la enfermedad después de descartar otros problemas de salud, que usualmente identifican tras valoraciones físicas y exámenes especializados. Por su parte, la valoración psicológica trata de abordar todas las áreas personales de la persona consultante, de modo que se brinde una atención integral a su condición de salud general, tal y como sugiere la guía de excelencia para la atención clínica en trastornos de dolor neuropático en adultos de NICE (2020b). La terapia cognitivo-conductual fue el principal enfoque de intervención psicológica que se encontró tanto en la literatura como en los resultados de este estudio, debido a la importancia de tratar los síntomas asociados que se presentan en esta población (Villanueva et al. 2004, Pikoff 2010).

Con respecto al tratamiento médico, la indicación de antidepresivos y analgésicos por parte del especialista entrevistado también coincide con la literatura revisada (Rivera 2008, Burke et al. 2012). Acerca de esto, cabe mencionar también la coincidencia en los 


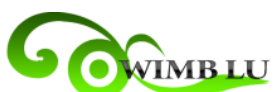

Wimblu, Rev. Estud. de Psicología UCR, 16(1) 2021 (Enero-Junio): 121-138 /ISSN: 1659-2107

resultados sobre la intervención de otras áreas, tales como la terapia física y la indicación de realizar natación y ejercicios de bajo impacto (Ubago et al. 2005). Si bien los resultados no arrojaron datos de cómo se procede en centros de salud privados, se encontró que tanto el reumatólogo entrevistado como otros colegas que trabajan en el área privada recetan Cymbalta (antidepresivo) y Dium (proporciona efectos analgésicos, antiepilépticos y antidepresivos), medicamentos que han mostrado gran efectividad en el alivio de síntomas físicos de pacientes con fibromialgia.

\section{Referencias bibliográficas}

Batmaz, Ibrahim, Sariyildiz, Mustafa Akif, Dilek, Banu, Inanir, Ahmet, Demirkan, Zeynep, Hatipoglu, Namik, Atar, Murat y Cevik, Remzi. "Sexuality of men with fibromyalgia: what are the factors that cause sexual disfunction?". Rheumatol Int 33 (2013): 1265-1270. doi: 10.1007/s00296-012-2567-1.

Boomershine, Chad. "A Comprehensive Evaluation of Standardized Assessment Tools in the Diagnosis of Fibromyalgia and in the Assessment of Fibromyalgia Severity". Pain Research and Treatment 2012 (2011): 1-11. doi:10.1155/2012/653714 http://downloads.hindawi.com/archive/2012/653714.pdf

Burke, James, Sanchez, Robert, Joshi, Ashish, Cappelleri, Joseph, Kulakodlu, Mahesh y Halpern, Rachel. "Health care costs in patients with fibromyalgia on pregabalin vs. duloxetine". Pain Practice 12, n. ${ }^{\circ} 1$ (2012): 14-22. doi: 10.1111/j.15332500.2011.00470.x.

Caja Costarricense del Seguro Social (2017). "Egresos según diagnóstico principal 2017”. En Anuario Estadístico 2017. Costa Rica: Caja Costarricense del Seguro Social, 2017. https://www.ccss.sa.cr/arc/estadisticas/salud/80/an_est_c066c_2017.zip

Escudero-Carretero, María José, García-Toyos, Noelia, Prieto-Rodríguez, María Ángeles, Pérez-Corral, Olivia, March-Cerdá, Joan Carles y López-Doblas, Manuela. "Fibromialgia: percepción de pacientes sobre su enfermedad y el sistema de salud. Estudio de investigación cualitativa". Reumatología clínica 6, n. ${ }^{\circ} 1$ (2010): 16-22. doi: 10.1016/j.reuma.2009.04.008 https://www.reumatologiaclinica.org/es-pdf-S1699258X09001570

Faro, Mónica, Sáez-Francàs, Naia, Castro-Marrero, Jesús, Aliste, Luisa, Collado, Antonio y Alegre, José. "Impacto de la fibromialgia en el síndrome de fatiga crónica". Medicina Clínica 142, n. ${ }^{\circ} 12$ (2014): 519-525. doi: 10.1016/j.medcli.2013.06.030

García-Martín, Elena, García-Campayo, Javier, Puebla-Guedea, Marta, Ascaso, Francisco, Roca, Miguel, Gutiérrez-Ruiz, Fernando, Vilades, Elisa, Polo, Vicente, Larrosa, Jose, Pablo, Luis. y Satue, María. "Fibromyalgia is correlated with retinal nerve fiber layer thinning". Plos One 11, n. ${ }^{\circ}$ (2016): 1-15. doi: 10.1371/journal.pone. 0161574

https://journals.plos.org/plosone/article/file?id=10.1371/journal.pone.0161574\&ty pe=printable 
Geenen, Rinie y Bijlsma, Johannes. "Deviations in the endocrine system and brain of patients with fibromyalgia: Cause or consequence of pain and associated features?". Annals of The New York Academy of Sciences 1193, n. ${ }^{\circ}$ (2010): 98-110. doi:10.1111/j.1749-6632.2009.05290.x

Ghib, Linda-Jéssica, Muntean, Laura, Tamas, LMaría-Magdalena y Rednic, Simona. "Concomitant Fibromyalgia Rheumatoid Arthritis: Challenges in Diagnosis and Disease Assesment”. Romanian Journal of Rheumatology 25, $\mathrm{n}^{\circ} .3$ (2016): 147-154. https://rjr.com.ro/concomitant-fibromyalgia-in-rheumatoid-arthritis-challenges-indiagnosis-and-disease-assessment/

Gómez-Argüelles, Jose María y Anciones, Buenaventura. "Prevalencia de síntomas neurológicos asociados a la fibromialgia". Revista de la Sociedad Española del Dolor 16, n. ${ }^{\circ}$ (2009): 222-229. doi: 10.1016/S1134-8046(09)71852-X http://scielo.isciii.es/pdf/dolor/v16n4/original3.pdf

National Institute for Health and Care Excellence "Chronic pain: assessment and management. In development [GID-NG10069]", 3 de diciembre del 2020(a). https://www.nice.org.uk/guidance/indevelopment/gid-ng10069

National Institute for Health and Care Excellence. "Neuropathic pain in adults: pharmacological management in non-specialist settings. NICE clinical guideline", 3 de diciembre del 2020(b). https://www.nice.org.uk/guidance/cg173/resources/neuropathic-pain-in-adultspharmacological-management-in-nonspecialist-settings-pdf-35109750554053

Parra, Marta, Latorre, José Miguel y Montañés, Juan. "Terapia cognitiva basada en mindfulness y reducción de los síntomas de ansiedad en personas con fibromialgia". Ansiedad y Estrés 18, n. ${ }^{\circ 2}$-3 (2012): 141-154.

Pereanu, Marcel. "Fibromyalgia". Acta Medica Transilvanica II, n. ${ }^{\circ}$ (2014): 191-192. http://www.amtsibiu.ro/Arhiva/2014/Nr4-en/Pereanu-en.pdf

Pikoff, Howard. "A study in psychological mislabeling: The rise and (protracted) fall of psychogenic fibromyalgia”. International Musculoskeletal Medicine 32, n. ${ }^{\circ} 3$ (2010): 129-132. doi: 10.1179/175361410X12798116924336

Revuelta-Evrard, Eva, Segura-Escobar, Estefanía y Paulino-Tevar, Javier. "Depresión, ansiedad y fibromialgia". Revista de la Sociedad Española del dolor 17, n. ${ }^{\circ}$ (2010): 326-332. doi:10.1016/j.resed.2010.07.002 http://scielo.isciii.es/pdf/dolor/v17n7/revision1.pdf

Rico-Villademoros, Fernando y Calandre, Elena. "Fibromialgia: ¿Comorbilidad marcadora de vulnerabilidad?”. Medicina clínica 142, n. ${ }^{\circ} 12$ (2014): 538-539. doi: 10.1016/j.medcli.2013.09.030

Rivera, Javier. "Tratamiento farmacológico de la fibromialgia". Información terapéutica del Sistema Nacional de Salud 32, n. ${ }^{\circ} 4 \quad$ (2008): 107-115. https://www.mscbs.gob.es/biblioPublic/publicaciones/recursos_propios/infMedic/ docs/vol32_4TratFarmFibromialgia.pdf

Rodríguez, Ricardo. "Identificación de Factores Psicológicos Asociados a la Activación ya la Inhibición se la Sintomatología Clínica de la Fibromialgia”. Acta Colombiana de Psicología 7 (2002): 85-102. 
Sallinen, Merja, Kukkurainen, Marja Leena, Peltokallio, Liisa y Mikkelsson, Marja. "Women's narratives on experiences of work ability and functioning in fibromyalgia". Musculoskelet 8, n. ${ }^{\circ} 1$ (2010): 18-26. doi: 10.1002/msc.162

https://actacolombianapsicologia.ucatolica.edu.co/article/view/1262/1196

Ubago, María del Carmen, Ruiz, Isabel, Bermejo, María José, de Labry, Antonio y Plazaola, Juncal. "Características clínicas y psicosociales de personas con fibromialgia: Repercusión del diagnóstico sobre sus actividades”. Revista Española $\begin{array}{lllll}\text { de } \quad \text { Salud } \quad \text { Pública } & \text { 79, } & \end{array}$ http://scielo.isciii.es/pdf/resp/v79n6/original5.pdf

Villanueva, Vicente Luis, Valía, Juan Carlos, Cerdá-Olmedo, Germán, Dolz, Monsalve, Bayona, María José y de Andrés, José Antonio. "Fibromialgia: diagnóstico y tratamiento. El estado de la cuestión”. Revista de la Sociedad Española del Dolor 11, n. ${ }^{\circ} 7$ (2004): 50-63. http://scielo.isciii.es/pdf/dolor/v11n7/revision2.pdf

Weber, Augusto, Werneck, Lineu, Paiva, Eduardo y Gans, Paulo. "Effects of Music in Combination with Vibration in Acupuncture Points on the Treatment of Fibromyalgia". The Journal of Alternative and Complementary Medicine 21, n. ${ }^{\circ} 2$ (2015): 77-82. doi: 10.1089/acm.2014.0199. 